$\begin{array}{ll} & \text { Etnográfica } \\ \text { etnográfica } & \text { Revista do Centro em Rede de Investigação em }\end{array}$

Antropologia

vol. $25(2) \mid 2021$

Vol. 25 (2)

\title{
Cuando ellas cuentan: relaciones de género y narrativas históricas sobre una antigua propiedad rural
}

When women tell: gender relations and historical narratives on an old rural property

Fernanda Figurelli

\section{(2) OpenEdition}

\section{Journals}

Edición electrónica

URL: https://journals.openedition.org/etnografica/9648

DOI: 10.4000/etnografica.9648

ISSN: 2182-2891

\section{Editor}

Centro em Rede de Investigação em Antropologia

Edición impresa

Paginación: 315-334

ISSN: 0873-6561

\section{Referencia electrónica}

Fernanda Figurelli, «Cuando ellas cuentan: relaciones de género y narrativas históricas sobre una

antigua propiedad rural», Etnográfica [En línea], vol. 25 (2) | 2021, Publicado el 29 julio 2021, consultado el 19 enero 2022. URL: http://journals.openedition.org/etnografica/9648; DOI: https://doi.org/ 10.4000/etnografica.9648

\section{(c) (1) (8)}

Etnográfica is licensed under a Creative Commons Attribution-NonCommercial 4.0 International License. 


\section{Cuando ellas cuentan: relaciones de género y narrativas históricas sobre una antigua propiedad rural}

\section{Fernanda Figurelli}

En este artículo me baso en un trabajo de campo realizado en las tierras de una gran propiedad rural del Nordeste de Brasil, hoy desaparecida. Parto de la historia sobre dicha propiedad que me cuentan antiguos habitantes del lugar que aún viven allí. Me interesa mostrar que esa historia, tanto en su contenido como en las dinámicas sociales de su narración, deja fuera las vivencias y los relatos de las mujeres. También las cuestiones académicas que llevo al trabajo de campo son asociadas con experiencias fundamentalmente masculinas. En el artículo exploro las narrativas que quedan fuera de la historia. En éstas asoman vivencias específicas de género, las cuales ocupan una posición marginal en los ámbitos enunciativos del lugar.

PALABRAS CLAVE: historia, narrativas, género, categorías sociológicas, Nordeste de Brasil.

When women tell: gender relations and historical narratives on an old rural property - This article is based on a fieldwork carried out in the lands of a former rural property in Northeastern Brazil. I consider the history of this property as told by its old inhabitants, who still live there. I intend to demonstrate that the content as well as the social dynamics of their narrations leave out the experiences and the accounts of women. Also, the categories of social sciences that I take to the fieldwork are predominantly associated with masculine experiences. In this paper I explore the marginalized narratives that fall outside the history of this place. In these, specific gender experiences emerge.

KEYWORDS: history, narratives, gender, sociological categories, Northeastern Brazil.

FIGURELLI, Fernanda (ferfigus@yahoo.com.ar) - Consejo Nacional de Investigaciones Científicas y Técnicas (Conicet), Argentina. 
POR MOTIVO DE MIS PREGUNTAS SOBRE UNA PROPIEDAD RURAL YA desaparecida, llamada "Belém", fuimos con Teresinha a Moreno, hacia la casa de su tío Joca, el hermano de su padre. ${ }^{1}$ Teresinha fue mi principal anfitriona durante el trabajo de campo. No fuimos por la ruta, como lo haría quien estuviera con automóvil, sino por un camino interno, más corto y adecuado para la caminata. La senda es usada por quienes ya la conocen. Los surcos que se abren en el pasto y algunos matorrales no se encuentran claramente marcados, de modo que no es difícil errar la vía, como de hecho nos ocurrió cuando intentamos un atajo. Si las personas se transportan en moto o caminando suelen tomar este sendero que une el asentamiento (assentamento) en el cual me hospedé para hacer mi trabajo de campo con la comunidad Moreno, lugares que antiguamente pertenecían a Belém. ${ }^{2}$

Caminamos un largo rato y en esa caminata atravesamos algunas parcelas donde las familias cultivan sus alimentos, llamadas rozados (roçados), y saludamos a habitantes de Moreno y del asentamiento que cruzábamos de cerca o que eran reconocidos por Teresinha a una gran distancia.

La lluvia nos acompañó casi todo el día y el trayecto no fue sin avatares. A pesar de las preocupaciones que aquello le generó a Teresinha, cuando el día se durmió, mi acompañante expresó que si pudiera volver a vivirlo lo haría. Teresinha se mostraba contenta de haberme ayudado en mi trabajo, presentado a sus parientes que viven en Moreno y mostrado cosas importantes de su vida a medida que transitábamos por el lugar.

En Moreno nos detuvimos en la mayoría de las casas por donde pasábamos, las cuales eran de parientes de Teresinha. Saludamos a una prima, a los sobrinos, al tío Joca, al hermano Biu y a la hija de la hermana. La hermana de Teresinha vive muy próxima de Biu y al lado de las ruinas de la casa de sus padres, quienes vivieron allí el último trecho de sus vidas.

En la casa de Biu, el hermano menor de Teresinha, estuvimos durante un tiempo prolongado. Almorzamos, conversamos, fuimos al rozado. Teresinha volvió al asentamiento con una bolsa de porotos que Biu le regaló y que recogieron juntos en el rozado de este último. Luego pasamos a visitar a la hermana de Teresinha, pero en su ausencia conversamos brevemente con la hija y volvimos a lo de Biu.

Una sutil diferencia se revela fundamental. Con Teresinha visitamos a sus parientes, a varios de ellos. Algunas de las visitas fueron breves, otras extensas. Sin embargo, no todas las visitas podían derivar en entrevistas. Había una

l En este artículo todos los nombres de personas y la mayoría de los nombres de lugares fueron cambiados. Las traducciones del portugués de entrevistas y conversaciones fueron hechas por la autora. Agradezco los comentarios de los/as evaluadores/as anónimos/as.

2 Los asentamientos de reforma agraria son tierras desapropiadas por el Estado para morada y trabajo agrícola de familias sin propiedad. Surgen en gran medida a partir de ocupaciones de tierras improductivas hechas por trabajadores y trabajadoras rurales. 
distinción implícita entre conocer la vida de Teresinha (y de las otras personas que vivieron mucho tiempo en el lugar) y hacer una entrevista sobre Belém. Teresinha sabía que me interesaba todo aquello, pero para ella todo aquello no era lo mismo. Guiada por Teresinha en Moreno, sólo hablé de Belém con su tío Joca Souza. Sólo a él le hice una entrevista y sólo con él encendí el grabador. A pesar de que también quise entrevistar al hermano menor de Teresinha, esta idea no gozó de gran repercusión y éste permaneció en el ámbito de las visitas. Así, se distinguieron dos circuitos: visitar a los parientes y conocer la vida de alguien que había pasado mucho tiempo en el lugar tomaba un significado diferente al de entrevistar a los antiguos y conocer la historia del lugar.

Joca Souza era el más viejo, él sabría hablarme de Belém. Su género y edad lo habilitaban más que a los otros a transmitirme aquella historia. Cuando llegamos, Joca estaba sentado en una piedra, debajo del anacardo del fondo de su casa. Más tarde, además de Joca, estábamos su hija que vive en Natal y lo visitaba, Teresinha y yo. Allí estuvimos algunas horas, allí lo entrevisté.

"Si me preguntas, tengo 87 años, sé de todo", me dijo Joca, y Teresinha dispuso las pautas de la entrevista de acuerdo a lo que dedujo que era de mi interés. Las preguntas de Teresinha en esa entrevista son de un gran valor a la hora de entender un sentido común sobre lo que Belém significa entre los antiguos trabajadores del lugar. Y de mayor valor aquí es el hecho de que, aunque la mayoría de las respuestas eran conocidas por ella, quien las respondía y, en consecuencia, contaba la historia de Belém, era su tío. Más adelante, cuando entrevisté a Teresinha, su relato no se centró sobre estas preguntas.

En base a un trabajo de campo realizado en las tierras de la antigua propiedad, en este artículo me detengo en los relatos de las mujeres que habitan allí y su relación con la historia de Belém. Me interesa mostrar cómo aquello que se produce como "histórico" por los habitantes del lugar deja fuera las experiencias y las narrativas de las mujeres. ${ }^{3}$

En este sentido, miro hacia un vacío percibido por diferentes autores y autoras que, ante el mismo, señalan la necesidad de dar una atención específica a las vivencias de género (con las críticas a la noción de cultura que eso trae aparejado). Abu-Lughod (1999) se desvía de la ideología central de tribus beduinas del desierto occidental de Egipto y se enfoca en los ghinnāwas, poemas líricos que son cantados en situaciones íntimas por mujeres y hombres jóvenes, que son centrales en la vida cotidiana y que niegan el código moral oficial del honor y la modestia.

La cuestión se destaca en los trabajos que se agrupan en la primera parte de la compilación de Appadurai, Korom y Mills sobre narrativas y género en

3 Si bien es el aspecto que enfoco aquí, las desigualdades que permean esa historia no nos remiten únicamente al género. Clase, posición social y edad ocupan un lugar central en los mecanismos mediante los cuales se construyen las diferentes historias que me contaron en el campo (ver Figurelli 2011, 2015). 
el Sudeste asiático (1991), los cuales se centran en enunciaciones de mujeres que manifiestan una especificidad no asimilable a los modelos oficiales. Tres de esos trabajos son enfáticos en este sentido. Ramanujan (1991) atiende los relatos de mujeres de Karnataka, India, y observa en ellos aspectos constitutivos de un sentido común que se opone a la tradición clásica de la India, un "contrasistema" que muestra que el mundo de las mujeres no es el mismo que el de los hombres. También focalizada en narrativas de mujeres, Grima (1991) revé el código de honor y modestia entre los pastunes. A partir de las diferencias que observa, la autora es explícita en la necesidad de no subsumir la cultura de las mujeres a los modelos masculinos dominantes, éstos últimos privilegiados en los estudios hechos por autores hombres (quienes han realizado la mayor parte de los estudios en el lugar). Por su parte, a diferencia de otros investigadores en Afganistán, que señalan una falta de participación de las mujeres en las narrativas orales, Mills (1991) considera los relatos de mujeres en el área de Herat y apunta a las distinciones (y desigualdades) que los mismos presentan con los relatos de los hombres. La autora subraya la diversidad cultural en las tradiciones expresivas afganas, la cual no puede apreciarse si, entre otras cosas, no se toma en cuenta el género. Las mujeres raramente performan para adultos que no sean miembros de la familia. De modo que la apreciación de los otros investigadores puede deberse a una falta de oportunidad en la observación (Mills 1991). ${ }^{4}$

La crítica a una epistemología que excluye sistemáticamente las experiencias de las mujeres o, de un modo más apropiado, de quienes han desempeñado roles socialmente construidos como femeninos, ha sido un punto de partida en trabajos en torno al género y la historia, así como al género y la memoria. La historia oral se destaca en este sentido. En esos trabajos ha primado la búsqueda por "dar voz" a las mujeres e incorporar sus narrativas y experiencias a la investigación científica. La bibliografía es variada, vasta y proviene de diferentes disciplinas (ver, por ejemplo, Woortmann 1992; Barrancos 2004; Perrot 2008; Jelin 2011; Passerini 2016; Leydesdorff, Passerini y Thompson 2017 [1996]; o Rooney 2008, quien revisa la bibliografía desde el análisis interseccional).

La necesidad de echar luz sobre lo que a simple vista no se ve, sobre lo que no se cuenta como historia, sobre las voces de quienes no cuentan la historia y quedan fuera, es también un punto de partida en este artículo. Pero más que incorporar voces a relatos históricos o registrar las formas que toman las

4 También Gold (1991) vislumbra una diversidad cultural con base en el género al analizar una versión regional de un relato clásico de la India que, además de tener sus fuentes en la tradición yogui, se impregna de narrativas regionales de mujeres. En la misma compilación, Claus (1991) se centra en una tradición oral cantada de habitantes de la costa de Karnataka, focaliza en cantos de mujeres y apunta a las especificidades que éstos presentan. 
memorias, como es, éste último, un camino común en los estudios de memoria, me interesa hacer hincapié en los mecanismos sociales por los cuales algunas voces - las de las mujeres en el caso que aquí nos ocupa - son excluidas de la producción histórica en el lugar. En vez de sumar relatos callados, de traer nuevos contenidos a la descripción de un pasado pretendidamente inclusivo, quiero enfatizar algunas dinámicas concretas de conformación y circulación de narrativas históricas, particularmente aquellas que producen desigualdades entre lo que hombres y mujeres cuentan.

Atender narraciones marginales me permitió vislumbrar las relaciones de género que en torno al relato de la historia de Belém autorizan las voces masculinas para contarla, para hablar en público y para ser grabadas y registradas. Si los hombres pueden contar la historia, las mujeres, en cambio, cuentan su vida, su historia o la historia de su vida. Los hombres también pueden contar su vida, pero las mujeres no pueden contar la historia. Así, lo que las mujeres cuentan toma un nombre diferente (y menos trascendente para las categorías de las ciencias sociales) de lo que los varones cuentan. Sus voces resuenan además en situaciones predominantemente informales (a excepción de algunos rituales eclesiásticos) y ante otras mujeres u hombres más jóvenes, sean vecinos, familiares, parientes o quienes tienen con ellas alguna relación personal, mientras que los hombres también son oídos por las mujeres de su generación y por posibles desconocidos o desconocidas que, posiblemente, expandirán lo escuchado en una geografía más amplia.

Así, las mujeres no hablan de la historia de Belém, ni tampoco son recomendadas para hacerlo. Mis preguntas, que giraban primero alrededor de un conflicto sindical ocurrido previamente a la dictadura militar, y después en torno a Belém, me fueron conduciendo por una red de recomendaciones que me destinaba a relatos masculinos, y si no hubiera sido por los avatares del trabajo de campo y por los imponderables que ese trabajo nos permite apreciar, el resultado final no hubiera tenido en cuenta la experiencia de las mujeres. En este sentido, además de mirar las dinámicas sociales por detrás de lo que se cuenta, específicamente aquí las relaciones de género, en este artículo remarco que también nuestras preguntas de investigación deben ser revisadas. La cuestión que me guió a las tierras de Belém iluminaba temáticas centrales a la ciencia social, entre éstas el abordaje del conflicto social; sin embargo, me remitía a experiencias predominantemente masculinas. Si me hubiera contentado con aquello, tal vez hoy estaría escribiendo sobre el conflicto de un antiguo latifundio y sobre la narración de ese conflicto por parte de los explotados, pretendiendo con ello dar cuenta de experiencias tanto de hombres como de mujeres. Aquí subrayo la necesidad de tener precauciones, no únicamente las relaciones de género en el lugar donde hacemos el trabajo de campo, sino también las relaciones de género que enmarcan nuestras propias preguntas de investigación afectan los análisis etnográficos. 


\section{UNA HISTORIA PARA CONTAR}

Emprendí mi trabajo de campo interesada en las diversas formas de aprehensión de un conflicto ocurrido al sur de Rio Grande do Norte, Brasil, en la región agreste, subregión del Nordeste brasileño, cuya diversidad de paisajes muestra una transición entre la zona húmeda de la costa y la zona seca del interior (Andrade 1998). Belém era una gran propiedad rural, una fazenda, en la cual se criaba ganado y se producía algodón. Se encontraba habitada por el dueño (el fazendeiro), su familia y por los moradores y sus familias. Éstos últimos constituían su fuerza de trabajo. En la propiedad recibían casa, la cual incluía la tierra para plantar los productos destinados a la subsistencia familiar. En menor medida también criaban animales para el autoconsumo. Aquello suponía su contrapartida: la diária, que consistía en un día por semana de trabajo gratis para el propietario, y el foro, definido por los ex moradores como un arrendamiento que se pagaba anualmente. Además debían plantar algodón, el cual constituía un cultivo comercial, pero que, de forma obligatoria y en condiciones desventajosas, tenía que ser vendido al dueño de las tierras. ${ }^{5}$

El territorio abarcado por la propiedad se divide actualmente en siete municipios. Una parte importante está ocupada por comunidades constituidas en gran medida por ex habitantes de Belém y sus descendientes, quienes compraron pequeñas parcelas en el lugar. En el presente, se dedican predominantemente a tareas agrícolas y/o venden su fuerza de trabajo en diversos empleos. Desde el año 2001 también existe allí el asentamiento de reforma agraria, resultante de una ocupación de tierras organizada por el sindicato de trabajadores rurales de Bom Jesus (éste último uno de los municipios donde la propiedad se extendía), en el que también viven antiguos habitantes de Belém. Como mencioné al inicio del artículo, en ese lugar, y específicamente en la casa de Teresinha y su esposo Gregório, me hospedé para realizar parte de mi trabajo de campo. Ambos habían nacido y vivido toda la vida en aquella región. Gregório era en ese momento presidente del sindicato mencionado. Con él establecí mi primer contacto en las tierras de la vieja propiedad, ayudada por la Federação dos Trabalhadores na Agricultura do Estado do Rio Grande do Norte (Fetarn), que se situaba en la capital de ese estado. Los integrantes de la Fetarn fueron mi relación inicial en Rio Grande do Norte.

La actividad sindical que ocurrió en Belém en años previos a la dictadura militar en el país es destacada por integrantes y ex integrantes de la Federación, quienes presentan a Belém como un hito del sindicalismo rural en todo Brasil. En 1961 fue fundado el sindicato de trabajadores rurales en la ciudad

5 En la propiedad también había otros trabajadores que se posicionaban en una más alta jerarquía. Para fazendas de ganado en el Nordeste de Brasil, ver, entre otros, Cascudo (1956), Johnson (1971), Bastos (s.f.), Almeida y Esterci (1977a, 1977b). 
de Bom Jesus (localizada en el municipio homónimo), y luego, la delegacia sindical de Belém. El sindicato fue reconocido por el Ministerio de Trabajo en 1962 (periódico A Ordem 1962), año en que también fue fundada la Fetarn, la cual nucleó a los sindicatos. En Rio Grande do Norte la iglesia católica imperó entre las demás fuerzas políticas que se disputaban la hegemonía en el campo, entre éstas la Liga Camponesa y el Partido Comunista Brasileiro (Cruz 2000). El sindicato de Bom Jesus se cuenta entre los primeros surgidos del trabajo de sindicalización rural emprendido por personas vinculadas a la iglesia.

Cuando los integrantes de la Federación narran las experiencias sindicales de ese momento, destacan un episodio al que se refieren como el "derrumbe del cambão" "derrubada do cambão"). Dicho episodio alude al fin del trabajo gratuito que los moradores realizaban para el propietario (la diária, como denominada por los habitantes del lugar). ${ }^{6}$ Belém era una de las más grandes propiedades en Rio Grande do Norte que en aquel tiempo mantenía el sistema de cambão y su rechazo por parte de los trabajadores sindicalizados tuvo gran importancia en la organización. Durante la dictadura militar el sindicato no se cerró, pero enfrentó grandes limitaciones, además sus integrantes fueron perseguidos, encarcelados y otros debieron exiliarse.

Me enteré del evento por el relato del primer presidente de la Federación de los Trabajadores que había participado de la organización sindical en aquella región. Ese relato llegó a mí por medio del proyecto "Memória Camponesa e Cultura Popular" (Memoria Campesina y Cultura Popular), con sede en el Núcleo de Antropologia da Política-NuAP, Museu Nacional, Universidade Federal do Rio de Janeiro, Brasil. ${ }^{7}$

Con el interés de indagar las maneras en que las personas relacionadas con el "derrumbe del cambão" reconstruían ese episodio, comencé por considerar las perspectivas que vislumbré desde mi lugar en la universidad: las de quienes se habían destacado en las experiencias sindicales de la década del 60. Durante el trabajo de campo, realizado entre los años 2008 y 2010, las recomendaciones de personas para conversar sobre las cuestiones que yo preguntaba configuraron diversos circuitos de investigación que reformularon mis indagaciones.

Mis primeras preguntas fueron acerca del cambão y su fin. No obstante, en las tierras de la antigua propiedad me di cuenta que el "cambão" o "el conflicto

6 En la bibliografía, el cambão se define como el trabajo gratuito (o, de acuerdo con Andrade 1998, también a muy bajo precio) que los moradores debían dar al dueño de la propiedad en la cual residían como contrapartida de la tierra que ocupaban (Julião 1962, 1968; Azevedo 1982). Sobre la distinción entre cambão y condição, ver Palmeira (1977) y Sigaud (1979). La categoría es analizada en Figurelli (2017a).

$7 \quad$ El proyecto focaliza en las luchas campesinas previas a 1964 y durante el período de resistencia a la dictadura. En él participan diversas instituciones de investigación, organizaciones sociales y organismos estatales (disponible en < https:/memov.com.br/site/component/k2/item/74-acervo-de-memoria-camponesa-e-cultura-popular $>$, última consultación en junio 2021). 
de Belém” eran un rótulo más adecuado entre los integrantes y ex integrantes de la Federación y que una de las mejores formas de iniciar las conversaciones en el sur norteriograndense sería, en cambio, preguntando sobre la vivencia que mi interlocutor o interlocutora habían tenido en Belém. A partir de allí la "historia" - como fue llamada - comenzaba a abrirse o, en su defecto, la recomendación de quien pudiera contarla.

Esa historia que se abría en las tierras de la antigua propiedad era la de los esclavos (escravos) o cautivos (cativos). Dicho de modo breve, tales categorías refieren a las experiencias laborales de los moradores en relación con los patrones, las cuales evocan la idea de ser amarrado (Figurelli 201 1, 2017a). ${ }^{8} \mathrm{La}$ diária, el foro que pagaban anualmente por la tierra que habitaban y la venta de algodón son las prácticas mencionadas por quienes habitan la propiedad cuando aluden a la esclavitud. La imposibilidad de negarse a ellas es reiterada en uno y otro relato. Si no cumplían la obligación de ir a la diaria, los moradores eran llevados a trabajar por el capanga, amarrados en el rabo de una burra. En el caso de la venta de algodón, los vigías de la propiedad impedían que éste fuera llevado hacia afuera, por lo cual no había otra opción que venderlo desventajosamente en el almacén del propietario. Con ello también se pagaba el foro (y, posiblemente, las deudas). ${ }^{9}$

Cuando los ex moradores refieren a Belém hablan de un tiempo que hace discontinua la línea entre pasado y presente, un tiempo de esclavos. La esclavitud se constituye principalmente por las experiencias de los moradores, y son ellos quienes preferencialmente contarán la historia.

\section{¿QUIÉN CUENTA LA HISTORIA?}

Entre los/as habitantes de Belém emerge una historia antigua. Las personas más viejas - parientes, vecinos o amigos de quien recomienda -, fueron las indicadas para contarla. Correspondía narrar a quienes habían vivido la historia o a quienes la escucharon contar directamente por ellos/as. Además, las consideraciones sobre "saber contar una historia" eran parte de las indicaciones, como también los ideales morales y los modos de comportamiento que disponían que los varones fuesen quienes acostumbraban a hablar en situaciones formales con gente de afuera. La situación de entrevista y la palabra pública que ésta instituía eran preponderantemente asociadas a los hombres. Se estimaba que quien podría contar de forma más adecuada la historia del lugar a una entrevistadora que quería saber sobre aquello era un hombre y no una mujer. Aún

8 Sobre la noción de cativeiro entre poblaciones rurales del Nordeste de Brasil, ver Lopes (1978), Sigaud (1979), Garcia Jr. (1983), Velho (1995).

9 Sobre los mecanismos de endeudamiento en los sistemas de morada ver Palmeira (1979, s.f.); Bastos (s.f.). Éstos eran centrales para la retención de la fuerza de trabajo. 
repleta de cuestiones personales, la experiencia contada por los hombres era considerada de mayor interés público que la experiencia contada por las mujeres. Finalmente, si las personas de mayor edad eran las narradoras privilegiadas de una "Belém antigua", entre ellas se destacaban los varones, los que habían sido moradores y habían vivido de forma directa el cautiverio o la esclavitud.

Además de ser remitida a los hombres en la mayoría de los casos, si en las situaciones de entrevista era la pareja la que estaba presente, el hombre era quien tomaba la palabra y la mujer se limitaba a hacer intervenciones. Cuando el varón no estaba, las mujeres preferían decirme que volviera más tarde a entrevistar al marido. Expresaban que éstos o sus vecinos podrían explicarme mejor sobre temas referidos al sindicato. A su vez, luego de que me remitían a ellos, también fueron fluidas sobre esos asuntos. La mayoría de las conversaciones grabadas con mujeres pudieron ser realizadas por la ausencia de sus maridos y varias de ellas ocurrieron sólo a partir de mi insistencia en hacerlo.

La historia de Belém traía no sólo un contenido sino también las dinámicas sociales que legitimaban a sus narradores. Las relaciones de género y las normas de comportamiento femenino y masculino en el lugar afectaron directamente los relatos en torno a la historia.

Sin embargo, esa historia no fue la única narrativa que, a partir de mis preguntas y de mi presencia, se abrió entre los/as habitantes de Belém. Tuve un acceso importante a las mujeres y si bien las situaciones de entrevista fueron el primer paso en ese acercamiento, las relaciones más informales que establecí durante mi trabajo de campo fueron fundamentales. En esas relaciones mi posición de investigadora perdió su relieve y mi vínculo con las mujeres se consideró más apropiado que mi vínculo con los hombres. Correspondió que compartiera con ellas y con sus hijos/as pequeños/as los momentos que no me dedicaba a entrevistar, momentos en los que, de acuerdo con su opinión, yo no estaba trabajando. Teresinha me llenaba entonces de atenciones que giraban en torno a la comida y al descanso. Cada vez que volvía de una entrevista intentaba con sus cortesías hacer un lugar a mi pausa. Yo comenzaba entonces a escuchar frases como: "ahora vas a comer algo, no vas a hacer nada". Acorde con la reflexión de Mills (1991), cuando narra el modo en que las relaciones de género afectan profundamente la visión que una investigadora o un investigador se llevan de su campo de trabajo, esos momentos de "no hacer nada" me revelarían una sociabilidad femenina y una parte importante de las experiencias de las mujeres de Belém.

Con las mujeres también hacía "visitas" y "paseaba", ellas me "acompañaban” a diferentes sitios y por medio de esas prácticas podía circular por el lugar, conocerlo y conversar con sus vecinos y vecinas, sus amigos y amigas, sus parientes y familiares.

La relación próxima que establecí con las mujeres me permitió acceder a sus rutinas cotidianas y a sus relatos sobre su vivencia en el lugar. Esos relatos 
enfatizaban aspectos diferentes a la relación de los moradores con el propietario - entre otros elementos que constituían la historia de Belém -, y mostraban una notable recurrencia entre ellas. Las mujeres hicieron que me desplazara de un núcleo central a los márgenes de la historia para, finalmente, dar un paso fuera de allí.

$\mathrm{Al}$ inicio de este artículo, observamos la diferencia entre las entrevistas sobre Belém y las visitas a los parientes y vecinos, entre las narrativas sobre Belém y las narrativas sobre la vida de alguien del lugar. Una y otra eran cuestiones diferentes y me fui dando cuenta de aquello a medida que transcurría la investigación. Sólo después de un tiempo entendí lo que Teresinha quería significar cuando, refiriéndose a mi investigación, decía que yo "quería saber de todo". Teresinha me estimuló a recorrer diversos circuitos que en un comienzo yo no supe diferenciar.

Muy sutilmente, ella me introducía a la historia al mismo tiempo que me sacaba de allí y me mostraba que existían otros significados en juego. Cuando fuimos a Moreno, si bien nos detuvimos un largo rato en la casa de su tío Joca, porque así lo deseaba yo, ella propuso con mayor entusiasmo llevarme a la casa de su hermano, mostrarme la casa de sus padres, conversar con su familia y narrarme su vida. Con ella, el relato se desplazaba de las relaciones con el propietario a relaciones vecinales y parentales, de contar la historia a contar una vida.

Las mujeres no eran ajenas al tiempo de los esclavos ni a los demás elementos subsidiarios que hacían a la historia de Belém entre los ex moradores, ellas conocían y reconocían esa historia e incluso me ayudaban a mapear la investigación. Cuando les preguntaba, era posible ver en su narración una mirada sobre Belém que ponía en primer plano el sufrimiento que el régimen de la propiedad implicaba para los moradores y sus familias. ${ }^{10}$ Pero, como vimos, no eran ellas las contadoras recomendadas para hablar de ese asunto. Por lo general, tampoco era el tema que más les interesaba privilegiar en sus relatos.

En el tiempo de los esclavos que define la historia de Belém entre los habitantes de esas tierras, las mujeres ocupan un lugar secundario. Se considera que la esclavitud fue directamente vivida por padres, tíos, abuelos, maridos, en fin, por varones adultos. La experiencia de las mujeres al respecto es atendida en segundo plano. La esclavitud es una categoría fundamentalmente masculina y, en consecuencia, también lo es la historia de Belém que esa categoría define.

Aquello puso en cuestión mis propios intereses académicos y me mostró que todo lo que en ellos asumía rango de cuestión tenía que ver con la experiencia de los hombres. Los eventos que me interesaban: el trabajo en la propiedad para los patrones, el enfrentamiento sindical, el conflicto de Belém, 
en fin, "la" historia de Belém, me abrían un mundo esencialmente masculino. Desde las preguntas que mis categorías centrales a las ciencias sociales me llevaban a privilegiar no hacía más que atender a una parte de la experiencia de los hombres y hacer a un lado la experiencia de las mujeres, para las que no tenía cuestión.

La necesidad de Smith de partir de la experiencia femenina de todos los días para mirar a los sujetos de conocimiento como mujeres y hombres que tienen cuerpo, que conocen y sienten, que se localizan en un espacio y en un tiempo concretos; para mirar la ciencia social, sus conceptos, sus abstracciones, como prácticas socialmente organizadas y dominadas por un punto de partida masculino; en fin, la necesidad de hacer visible el proceso social de construcción de categorías sociológicas, de un conocimiento masculinamente centrado, tomó para mí en este contexto todo su sentido (Smith 1992; Collins 1992).

Describiré ahora las experiencias femeninas que la historia de Belém deja en un papel secundario. Circularé por los relatos que se fueron tejiendo un poco en las entrevistas, pero sobre todo en mi contacto más personal con las mujeres, a partir de mi estadía en la casa del asentamiento y de los "paseos" y "visitas" que hacía en las tierras de la antigua Belém, y observaré el modo en que las mujeres colocan en primer plano una narrativa diferente a la de la historia de Belém.

\section{MÁS ALLÁ DE LA HISTORIA}

\section{Una vida de trabajo}

Cuando abría mis conversaciones con los ex moradores y sus esposas al preguntar como era su vivencia en Belém, algunas respuestas señalaban el nacimiento y la permanencia en el lugar, "yo nací y me crié en Belém”, y luego se remitían a la propiedad, a la familia propietaria, a los capataces del propietario, o al tiempo de los esclavos, entre otros elementos de la historia que se significa como antigua. Otras respuestas, en cambio, no hablaban de una historia de Belém y establecían una continuidad, un eterno gerundio que versaba sobre el propio trabajo. Si bien con excepciones, es posible señalar una tendencia y decir que lo primero ocurría entre los hombres, mientras que lo segundo solía ocurrir entre mujeres. Cuando los hombres hablaban de su trabajo, tampoco dejaban de marcar una permanencia, si bien ya no era considerado una parte central del relato que hacían sobre Belém y su historia. Su trabajo en el rozado y con los animales era algo de siempre, aunque con cambios a través de los años. Me enfocaré en el relato de Teresinha.

Cuando conversaba con Teresinha y también cuando la entrevistaba, ella enfatizaba asuntos distintos de los que le preguntó a su tío Joca el día que fuimos a Moreno. La primera vez que le dije que quería "entrevistarla", me respondió que el mejor horario sería luego de la cena, cuando estaba más tranquila 
para "contarme su vida". ${ }^{11}$ Todas las veces que la "entrevisté" fueron en ese horario y en la galería cubierta que rodea la casa, cerca de la puerta de la cocina, donde hay una mesa en la que acostumbrábamos a comer, además de varias sillas confortables. También allí suelen colgar la hamaca para descansar. La segunda vez que la grabé comencé la conversación diciendo que me cuente, no de la historia de Belém, sino "de su vida", ya que era así como Teresinha había traducido mis preguntas en lo que a ella concernía. Me respondió con un canto:

"Una novela de tristeza y de ilusión

Parece que el destino quiso traicionarme

Pero mi esperanza está perdida

Si fuera a contar mi vida

Dolería en cualquier corazón

[Y rió, luego prosiguió:]

Ya amé, ya fui amada

Ya viví bien satisfecha

Nunca pensé que la nostalgia fuese a habitar mi pecho

Pero mi esperanza está perdida

Si fuera a contar mi vida

Dolería en cualquier corazón"

Como en la frase de la canción, Teresinha comenzó a "contarme su vida", en la que, como una "cosa buena" o como "sufrimiento", el trabajo toma un lugar de relieve. ${ }^{12}$ Desde muy pequeña, Teresinha planta y cosecha; en aquella época lo hacía con su familia, sus hermanos varones se dedicaban a tareas agrícolas diferentes:

"Así es, mi hija, así era nuestra vida. Qué cosa buena. De chica, yo trabajaba mucho en el rozado, con seis años ya estaba en el rozado, trabajando en la agricultura con mi padre y mis hermanos. Plantaba poroto mulatinho, plantaba un montón de papa, plantaba maíz, sembraba haba, plantaba haba, poroto, coseché mucho poroto verde, mucho poroto (mulatinho), mucho maíz, mucha haba, todo eso hacíamos." 13

11 Las conversaciones luego de la cena son corrientes en el lugar. Autores como Cascudo (1956) llamaron la atención sobre este hecho, que no se restringe a la región estudiada. Ver, por ejemplo, Heredia (1979).

12 En este apartado, todas las frases entre comillas, salvo aclaración, son frases de Teresinha.

13 Como observa Garcia Jr. (1983) para la Zona da Mata sur de Pernambuco y Heredia (1979) para la Zona da Mata norte, entre pequeños productores la preparación de la tierra es una tarea considerada masculina, como también la realización de aberturas para depositar las semillas. El depósito de las semillas, la plantación, es una actividad propia de las mujeres. 
Por la noche, a las tres o cuatro de la madrugada, Teresinha salía de su casa con su padre y sus hermanos para trabajar en el rozado. Llegaban allí y comenzaban a recoger habas, a cortar maíz, a recoger algodón. Antes de partir al rozado comían harina de maíz que el padre molía en el mortero con rapadura. Cuando volvían del trabajo cenaban, comían en platos de barro: "entonces lavaba todo, sin jabón, así nomás, comía de nuevo, servía de nuevo harina de maíz, mungunzá, así era la vida".

Además de trabajar en el rozado del padre, Teresinha tuvo un roçadinho (rozadito), que era su plantación individual. ${ }^{14}$ El roçadinho dependía de ella, la única integrante de la familia a quien el padre le había dado uno. A las cinco de la tarde dejaba de trabajar en el rozado del padre y se iba a su roçadinho. Iba a buscar agua para regar sus plantaciones. Cuando llovía, el agua se acumulaba en un estanque cerca de su casa y el trayecto se le hacía más corto que cuando no llovía, en ese caso Teresinha buscaba agua más lejos. Para eso debía atravesar los cercados del dueño de la tierra, que no quería que las personas pasaran por allí porque los alambres y las estacas se ablandaban.

“- ¿Quién no quería?" [La interrumpí para preguntarle un dato que para ella no era relevante].

- "Antonio Melo" [éste era el nombre de un miembro de la familia propietaria de Belém] - me respondió, y continuó. Llevaba el agua en la cabeza y pasaba todos los alambres para poder regar su roçadinho. Teresinha iba acompañada de su hermano Biu (de Moreno), que era seis años más joven que ella. El padre no la dejaba andar sola, pero sí la dejaba con Biu.

Así fue la vida durante tres años, desde sus quince años, cuando su padre le dio el roçadinho, hasta que se casó y fue a trabajar con su esposo Gregório. Entonces continuó "la misma vida", siguió trabajando, como siempre lo hizo: "con mi papá, cuando me casé, siempre seguí trabajando". Teresinha tuvo ocho hijos y trabajó mucho para cuidar de ellos/as, de día y de noche. De día trabajaba en el rozado, plantaba, cosechaba, limpiaba el matorral. Antes de ir hacia allí dejaba las tareas listas: las habas cocinadas, el maíz para las gallinas y luego partía. El rozado era lejos y la esperaba media hora de camino. A medida que llegaba a su destino, el sol salía con ella y cuando el sol se dejaba ver entero, ella ya estaba allí. Era un rozado de porotos, de habas, de algodón, de maíz, de mandioca, de papa.

Si cuando era pequeña Teresinha "vivía en el rozado", después que se casó sus actividades se ampliaron. De día trabajaba en el rozado y de noche y de madrugada se hacía cargo de la casa y de los animales que criaba. Lavaba ropa, alimentaba a los cerdos y a las gallinas y ordeñaba a las cabras. Tareas, objetos,

14 En los trabajos ya citados, Heredia (1979) y Garcia Jr. (1983) señalan que esa plantación individual puede pertenecer a cualquier miembro de la familia que no sea el padre, quien tiene el dominio del rozado. 
palabras, seres y relaciones constituyen la casa, la cual es una categoría central en las narrativas de las mujeres. Como algunos trabajos recientes dejan observar, la atención a aquello que la compone edifica en las ciencias sociales un acceso a sus experiencias (ver, por ejemplo, Borges 2013; Carneiro 2015; Figurelli s.d.; Guedes 2017; Dainese, Carneiro y Menasche 2018).

Cuando era noche de luna barría el patio. "Yo sufrí mucho en mi vida, ¿sabes?". El trabajo era para Teresinha "una belleza", pero también la hacía sufrir, trabajar mucho (y otros episodios de su vida), la llevaba a conformar la idea de una vida sufrida, de una vida de trabajo, "llena de sufrimiento y tristeza", de una vida en la que se doblegó para dar cuenta de la casa, para cuidar de sus hijos, para poder mandarlos a la escuela: "trabajé como un animal, como un burro, para atender los ocho hijos que tengo, para atender la casa, los animales que criaba, el rozado, todo, día y noche". Aquí el trabajo se hace presente para dar cuenta del sufrimiento de la vida. La expresión del sufrimiento en el relato de Teresinha aparece por lo general luego de la mención a su casamiento, lo cual recuerda lo constatado por Grima (1991) entre las mujeres pastunes.

Teresinha preparaba las comidas, trituraba el maíz en el mortero y lo cocinaba: "hacía cuscuz, hacía mungunzá, hacía canjica, hacía pamonha, hacía mucho en la época, mi hija, le daba a la gente, las vecinas se llevaban todito, le daba a uno, le daba al otro". Su relato sobre esas actividades es extenso, las explica en detalles y para eso se vale tanto de las palabras como de la mímica, además de mostrarme algunas de las herramientas utilizadas en la realización de las mismas.

Como Teresinha, también las otras mujeres centraron su relato en las tareas que realizaron a lo largo de los años. El trabajo, en la casa y en el rozado, marca entre ellas una permanencia, fue toda la vida y continúa, no pertenece al tiempo de los esclavos si bien lleva una marca de éste. A través del trabajo, el pasado no se transforma en historia.

El trabajo fue un elemento central de aquello que las mujeres me contaban cuando les preguntaba sobre su vivencia en Belém. Éste marca una permanencia. Además, muestra sus espacios de sociabilidad, las tareas realizadas colectivamente y que implican una socialización en la vida del lugar, o aquellas a partir de las cuales las mujeres se relacionan con sus vecinas $\mathrm{u}$ otras personas que no son estrictamente del ámbito de su hogar (Figurelli 201 l, s.d.).

\section{La vida en la iglesia}

Como ocurre con el trabajo, también los encuentros religiosos son considerados temas dignos de narrarme cuando las mujeres se refieren a su vivencia en el lugar. Sus relatos los colocan en primer plano. Al contar sus experiencias religiosas, ellas revelan espacios de sociabilidad que también hablan de una permanencia. No se restringen al pasado, por el contrario, sus narrativas 
presentan a dichas experiencias en continuidad con el presente. Volvamos al relato de Teresinha.

Durante una cena con Teresinha, desde la televisión que estaba mirando su hijo Zé Paulo escuchamos una canción que a ella le recordó los terços de Monte Bravo, donde vivía cuando era pequeña. Los terços también suceden actualmente y gozan de gran concurrencia. Se realizan en el templo católico del asentamiento todas las noches de mayo, a excepción de los fines de semana. En su mayoría se reunen mujeres y rezan una parte del rosario (un tercio), además de cantar melodías religiosas y leer versículos de la Biblia. La llave de la capilla queda a cargo de tres mujeres que viven allí. En los terços de mayo que tuve la oportunidad de frecuentar durante el trabajo de campo y en las narrativas de las mujeres fue posible ver que gran parte de la dinámica religiosa recreada en el lugar era un espacio de encuentro y de dominio femenino.

Monte Bravo es una comunidad que se erige en las tierras de la antigua Belém y se encuentra en la ruta que antes era el camino central de la propiedad. Cuando se pasa por allí es posible distinguir a lo lejos la iglesia del lugar. "Pídele a Gregório que te muestre la iglesita", me sugirió Teresinha, y así lo hice cuando él me acercó por esa ruta hacia la ciudad de Bom Jesus. "Esa es la iglesia”, me dijo Gregório, y señaló con la mano una construcción más nueva de lo que yo había imaginado. Me explicó que la iglesia había sido restaurada, pero que era la más vieja de allí. El patrono era San Juan Bautista, me dijo Teresinha, y luego cantó una composición encabezada por los versos que siguen:

"San Juan Bautista, su casa huele

A clavos y rosas y flor de lima

Viva, viva, viva San Juan Bautista, viva..."

Teresinha se reconstruye cantando aquello desde que nacieron sus primeros dientes. La canción le recuerda al padre y a la madre, y a la gente que se reunía en la iglesia de Monte Bravo y cantaba. Y con todos, ella también lo hacía. Para Teresinha aquello era "mucha cosa, mucha cosa bonita, y el sol subiendo, y la felicidad, nostalgias de mi padre y de mi madre".

Todo ocurría en esa iglesia, que de acuerdo con Teresinha también era de Nuestra Señora de Belém: "Desde que nací que vi esa santita ahí, nunca se rompió, viejita ya, Nuestra Señora de Belém, tenía un bebé en el brazo, una corona en la cabeza". La estatuilla de la santa que aún se encuentra allí es la que estaba cuando la iglesia se ubicaba a la orilla del río. Teresinha estimaba que la estatuilla tiene más de un siglo. Antes de que ella naciera, había ocurrido una inundación y, junto con la iglesia, el agua se había llevado el cementerio y las casas de quienes vivían en esa zona. Las personas habían conseguido, sin embargo, rescatar a la santa. La iglesia fue luego reconstruida en Monte Bravo, que es el lugar donde hoy se encuentra. 
“- Hoy pasé y la vi de nuevo - le comenté.

— ¿iViste la iglesia!? - Me respondió animada.

- Sí, ino te conté que la semana pasada le dije a Gregório que me muestre la iglesia!

— ¿iÉl te mostró!? ¿iLa viste!? ¿iLa viste!?

- La vi.

— iEsa ahí! La iglesita, chiquita, pero es bonita, Nuestra Señora de Belém y San Juan Bautista [...] Yo amo aquella iglesita de Monte Bravo, yo nací y me crié ahí, es chiquita, pero era mi felicidad."

Por su parte, el mes de junio se perfilaba para las fiestas de San Juan y traía consigo otra de las vivencias que me serían narradas. Habían comenzado los ensayos para los bailes y aquello se llevaba comentarios de varias de las mujeres con las que acostumbraba a estar. Al hablar de la fiesta de San Juan, la diversión y el entusiasmo se hacían presentes y se abría un espacio para que las de mayor edad contaran sobre esa fiesta que viene de larga data y que, me decían, es una "tradición". "Yo llevaba mis muñecas de trapo y jugaba", dijo Edna. Ya desde entonces las personas se bañaban en los embalses y cantaban: "San Juan, mi San Juan/Santo que todos veneran/Todos tienen su corona San Juan/Su capilla".

Quien siente dolor en el cuerpo o tiene alguna enfermedad, puede en el agua pedir a San Juan la cura: "San Juan, vine aquí para curarme”, me explicaron las mujeres. También se realiza una fogata, donde es posible asar el choclo para preparar la canjica y la pamonha. Además, la fogata permite dar luz al establecimiento de una relación de madrinazgo o de comadrazgo entre dos mujeres.

Y fue de este modo como, a partir de las relaciones más personales de mi trabajo de campo, se fue abriendo una porción de las experiencias de las mujeres de Belém que no se incluía en la historia.

\section{CONCLUSIONES}

Preguntar en torno al cambão, o al conflicto de Belém, no fue la mejor forma de iniciar las conversaciones con mis interlocutores e interlocutoras en las tierras de la antigua propiedad, de modo que comencé a preguntar sobre la vivencia en Belém. Con eso me adecué a ellos/as, o por lo menos a las generaciones más viejas, y abrí la puerta a una mayor cantidad de experiencias. No supe en ese momento que aquello no me permitía incluir los relatos de las mujeres sin subsumirlos a lugares secundarios. La pregunta por el cambão y el conflicto sindical, ajena a una gran parte de los pobladores y las pobladoras de la región, fue más tarde reemplazada por una cuestión que incluía el nombre del latifundio, el cual tenía una historia para ser contada más allá de la propia experiencia de quien la contara. La misma evocaba a los patrones y a la explotación que sufrían quienes trabajaban para ellos. Lo que no sabía entonces es que las 
mujeres no contaban la historia, y que la explotación que esa historia rescataba aludía predominantemente a experiencias masculinas.

De modo que, entre los habitantes de la región, Belém evoca una historia, una historia antigua, y esta autoriza a determinados narradores e instaura contadores privilegiados. A partir de mis preguntas, primero sobre el conflicto del fin del trabajo gratuito y después sobre las vivencias en Belém, fui siendo guiada - por hombres y mujeres - por un circuito de contadores de edad avanzada, predominantemente varones, que referían a un tiempo de esclavos, un tiempo de patrones que explotaban moradores. Las experiencias que componen ese tiempo refieren principalmente a la obligación de los hombres de trabajar gratuitamente en la diária o de entregar al propietario la totalidad del algodón que producían. Focalizan además en la relación entre trabajadores y patrones. Si bien las mujeres también trabajaban en el algodón, o sufrían con el trabajo que sus maridos o sus hijos hacían en la diária, su rol en esas experiencias queda relegado a un segundo lugar. Además, no eran ellas quienes se relacionaban directamente con los patrones. La historia que se abría ante mí era contada principalmente por hombres y aludía de forma primordial a sus experiencias. Las mujeres conocían y reconocían la historia pero no eran sus contadoras.

Tampoco las mujeres eran quienes de preferencia hablarían con alguien de afuera, que no era ni vecina, ni pariente, sino una investigadora de la universidad. Mi acceso principal a ellas fue a partir de las relaciones más personales que establecí durante el trabajo de campo, cuando "dejaba de investigar", cuando mi papel de "visita" tomaba protagonismo ante el papel de universitaria. Ellas no le contaban la historia a la investigadora (si bien la ayudaban con sus recomendaciones de personas para hablar); ellas atendían a la visita y le contaban, a la visita y a la investigadora, sobre su vida, o sobre la historia de su vida. Aún cuando mi pregunta remarcaba sobre la vivencia en Belém, esas vivencias no constituían la historia de esta última.

En ese relacionamiento con mujeres descubrí otra configuración de experiencias. Con ellas di un paso fuera de la historia y del circuito de recomendaciones y conseguí ver los límites de lo que los ex moradores cuentan sobre Belém. Las mujeres me revelaron las experiencias, no sólo individuales sino, y sobre todo, colectivas, que ellas hallan dignas de ser contadas a alguien que no es del lugar, y que, sin embargo, se cuentan en voz baja, por fuera de la narración de la historia. Las mujeres rememoran una experiencia continua; más que una historia con cierto grado de autonomía a ser narrada por expertos, cuentan sus propias experiencias de siempre. Así, las vivencias laborales y religiosas me permitieron ver una parte de aquello que había para contar y que no era una historia. De aquello que continuaba, que permanecía desde épocas antiguas. De aquello que hablaba de espacios de sociabilidad, pero que se contaba de forma silenciosa.

Esa constatación me obligó a revisar mis propias cuestiones, las cuales me habían llevado hacia las vivencias masculinas. Con mi rol de universitaria y 
con mis preguntas, surgidas del interés por los conflictos sociales, por los latifundios, por el trabajo para los patrones rurales o por los enfrentamientos sindicales, despertaba fundamentalmente las narrativas de los hombres. La historia dejaba fuera a las mujeres, pero la vida, el trabajo cotidiano, los "no-eventos", los espacios más ocultos de sociabilidad, las experiencias sin una clara clasificación académica que a veces quedan desatendidas cuando nuestros/as interlocutores/as en el campo las nombran, las traían. Las mujeres desplegaban modos alternativos de contarme sobre Belém y sobre la vida del lugar, modos que difícilmente se abrían con mis preguntas acerca de la historia y el conflicto, o el derrumbe del cambão, o la propiedad.

La historia que los habitantes de Belém producen pone al descubierto las relaciones de género por detrás de su producción. Asoman las dinámicas de sociabilidad y poder entre hombres y mujeres que la enunciación pone en juego y la inercia a la que pueden llevarnos algunas de nuestras preguntas de investigación. Aquello que se constituye como historia entre los habitantes de Belém y los rituales de la sociabilidad y del discurso que su narración exige dejan fuera las vivencias y los relatos de las mujeres. Sólo al dar un paso al costado de las narrativas consagradas y dar relevancia a las conversaciones que despuntaban en situaciones más personales vi que allí desbordaba un universo de experiencias que se contaba de modo marginal, que no era parte de la historia pero sí de una vida, la cual rescataba lo que las mujeres consideraban con valor para ser contado a alguien de afuera del lugar que se interesaba por aquello y que estaba de visita.

\section{BIBLIOGRAFÍA}

A ORDEM, 1962, "Reconhecidos oficialmente sindicatos rurais do RGN". Natal: Rio Grande do Norte, XX (5394): 8.

ABU-LUGHOD, Lila, 1999, Veiled Sentiments: Honor and Poetry in a Bedouin Society. Berkeley: University of California Press.

ALMEIDA, Alfredo Wagner Berno de, y Neide ESTERCI, 1977a, "Quixadá: A formação do povoado e o aceso à terra pelos pequenos produtores", Projeto Emprego e Mudança Sócio Econômica no Nordeste. Rio de Janeiro: Museu Nacional/UFRJ (mimeo), 2-24.

ALMEIDA, Alfredo Wagner Berno de, y Neide ESTERCI, 1977b, "Terras soltas e avanço das cercas", Projeto Emprego e Mudança Sócio Econômica no Nordeste. Rio de Janeiro: Museu Nacional/UFRJ (mimeo), 25-36.

ANDRADE, Manuel Correia de, 1998, A Terra e o Homem no Nordeste: Contribuição ao Estudo da Questão Agrária no Nordeste. Recife: UFPE. 
APPADURAI, Arjun, Frank J. KOROM, y Margaret MILLS (comps.), 1991, Gender, Genre, and Power in South Asian Expressive Traditions. Filadelfia: University of Pennsylvania Press.

AZEVEDO, Fernando Antonio, 1982, As Ligas Camponesas. Rio de Janeiro: Paz e Terra.

BARRANCOS, Dora, 2004, "Historia, historiografía y género: notas para la memoria de sus vínculos en la Argentina”, La Aljaba, IX: 49-72.

BASTOS, Eliane Cantarino O’Dwyer, s.f., A Cultura de Algodão no Sertão Paraibano. Mimeo.

BORGES, Antonádia, 2013, "Mulheres e suas casas: reflexões etnográficas a partir do Brasil e da África do Sul”, Cadernos Pagu, 40. Disponible en < https://doi.org/10.1590/S0104-8 $3332013000100006>$ (última consultación en junio 2021).

CARneiro, Ana, 2015, O Povo Parente dos Buracos: Sistema de Prosa e Mexida de Cozinha. Rio de Janeiro: e-papers.

CASCUDO, Luís da Câmara, 1956, Tradições Populares da Pecuária Nordestina. Rio de Janeiro: Ministério da Agricultura, Serviço de Informação Agrícola.

CLAUS, Peter, 1991, "Kin songs", in Arjun Appadurai, Frank, J. Korom, y Margaret Mills (comps.), Gender, Genre, and Power in South Asian Expressive Traditions. Filadelfia: University of Pennsylvania Press, 136-177.

COLLINS, Patricia Hill, 1992, “Transforming the inner circle: Dorothy Smith's challenge to sociological theory”, Sociological Theory, 10 (1): 73-80.

CRUZ, Dalcy da Silva, 2000, "Igreja católica no RN: participação política e social nos anos 60", en Ilza Araújo Leão Andrade (comp.), Igreja e Política no RN: Momentos de Uma Trajetória. Natal: Z Comunicação/Sebo Vermelho, 41-91.

DAINESE, Graziele, Ana CARNEIRO, y Renata MENASCHE, 2018, "Casa e corporalidade em contextos camponeses e de povos tradicionais”, Tessituras, 6 (2). Consultado en $<$ http:// dx.doi.org/10.15210/tes.v6i2.14792 > (última consultación en marzo de 2020, ya no disponible).

FIGURELli, Mónica Fernanda, 2011, Família, Escravidão, Luta: Histórias Contadas de Uma Antiga Fazenda. Rio de Janeiro: programa de posgrado en Antropología Social, Museu Nacional, UFRJ, tesis de doctorado.

FIGURELLI, Mónica Fernanda, 2015, "Histórias que são passadas: vozes e entidades que circulam nas reconstruções do passado de uma antiga fazenda", Mana, 21 (2). Disponible en <http://www.scielo.br/scielo.php?script=sci_arttext\&pid=S0104-9313 $2015000200347 \& \operatorname{lng}=$ en\&nrm $=$ iso $>$ (última consultación en junio 2021).

FIGURELLI, Mónica Fernanda, 2017a, "Atados al rabo de la burra: lecturas del trabajo gratuito en una antigua propiedad rural", Revista Latinoamericana de Antropología del Trabajo, l (2). Disponible en < http://www.ceil-conicet.gov.ar/ojs/index.php/lat/article/view/306 > (última consultación en junio 2021).

FIGURELLI, Mónica Fernanda, s.f., "Vivencias en voz baja: género, público y privado en poblaciones rurales de Rio Grande do Norte (Brasil)”, Cadernos Pagú (en prensa).

GARCIA JR., Afrânio Raul, 1983, Terra de Trabalho. Rio de Janeiro: Paz e Terra.

GOLD, Ann Grodzins, 1991, "Gender and illusion in a Rajasthani yogic tradition”, in Arjun Appadurai, Frank J. Korom, y Margaret Mills (comps.), Gender, Genre, and Power in South Asian Expressive Traditions. Filadelfia: University of Pennsylvania Press, 102-135.

GRIMA, Benedicte, 1991, "The role of suffering in women's performance of paxto", in Arjun Appadurai, Frank J. Korom, y Margaret Mills (comps.), Gender, Genre, and Power in South Asian Expressive Traditions. Filadelfia: University of Pennsylvania Press, 78-101. 
GUEDES, André Dumans, 2017, "Construindo e estabilizando cidades, casas e pessoas", Mana, 23 (3). Disponible en < http://www.scielo.br/scielo.php?script=sci_arttext\&pid=S0104 -93132017000300403\&lng=pt\&nrm=iso > (última consultación en junio 2021 ).

HEREDIA, Beatriz María Alasia de, 1979, A Morada da Vida: Trabalho Familiar de Pequenos Produtores do Nordeste do Brasil. Rio de Janeiro: Paz e Terra.

JELIN, Elizabeth, 2011 , "Subjetividad y esfera pública: el género y los sentidos de familia en las memorias de la represión”, Política y Sociedad, 48 (3). Disponible en < https://revistas. ucm.es/index.php/POSO/article/view/36420 > (última consultación en junio 2021 ).

JOHNSON, Allen W., 1971, Sharecroppers of the Sertão: Economics and Dependence on a Brazilian Plantation. Stanford, CA: Stanford University Press.

JUlião, Francisco, 1962, Que São as Ligas Camponesas? Rio de Janeiro: Civilização Brasileira. JULIÃO, Francisco, 1968, Cambão (le joug): La face cachée du Brésil. París: François Maspero.

LEYDESDORFF, Selma, Luisa PASSERINI, y Paul THOMPSON (comps.), 2017 [1996], Gender and Memory. Nueva York: Routledge.

LOPES, José Sérgio Leite, 1978, O Vapor do Diabo: O Trabalho dos Operários do Açúcar. Rio de Janeiro: Paz e Terra.

MILLS, Margaret, 1991, "Gender and verbal performance style in Afghanistan", in Arjun Appadurai, Frank J. Korom, y Margaret Mills (comps.), Gender, Genre, and Power in South Asian Expressive Traditions. Filadelfia: University of Pennsylvania Press, 56-77.

PALMEIRA, Moacir, 1977, "Casa e trabalho: nota sobre as relações sociais na plantation tradicional”, Contraponto, 2 (2): 103-1 14.

PALMEIRA, Moacir, 1979, "Desmobilização e conflito: relações entre trabalhadores e patrões na agro-indústria pernambucana”, Revista de Cultura e Política, l (1): 41-56.

PALMEIRA, Moacir, s.f., O Trabalho Livre nos Engenhos: Renda, Salário, Dívida. Mimeo.

PASSERINI, Luisa, 2016, "Una memoria para la historia de las mujeres: problemas de método e interpretación”, Aletheia, 7 (13). Disponible en < http://sedici.unlp.edu.ar/han dle/10915/58353 > (última consultación en junio 2021).

PERrot, Michelle, 2008, Mi Historia de las Mujeres. Buenos Aires: Fondo de Cultura Económica.

RAMANUJAN, Attipate Krishnaswami, 1991, “Toward a counter-system: women's tales”, in Arjun Appadurai, Frank J. Korom, y Margaret Mills (comps.), Gender, Genre, and Power in South Asian Expressive Traditions. Filadelfia: University of Pennsylvania Press, 33-55.

ROONEY, Eilish, 2008, "Critical reflections: documenting gender and memory", Women's Studies International Forum, 31 (6): 457-463.

SIGAUD, Lygia, 1979, Os Clandestinos e os Direitos: Estudo sobre Trabalhadores da Cana-de-Açúcar de Pernambuco. São Paulo: Livraria Duas Cidades.

SMITH, Dorothy, 1992, “Sociology from women's experience: a reaffirmation”, Sociological Theory, 10 (1): 88-98.

VELHO, Otávio, 1995, "O cativeiro da Besta-Fera”, in Besta-Fera: Recriação do Mundo. Ensaios Críticos de Antropologia. Rio de Janeiro: Relume-Dumará, 13-43.

WOORTMANN, Ellen, 1992, "O ambiente e a mulher: o caso do litoral do Rio Grande do Norte, Brasil”, Latin American Studies, 12: 30-53. 